\title{
ORIGINAL
}

\section{Critical care admission following elective surgery was not associated with survival benefit: prospective analysis of data from 27 countries}

\author{
Brennan C. Kahan ${ }^{1}$, Desponia Koulenti ${ }^{2,3}$, Kostoula Arvaniti ${ }^{4}$, Vanessa Beavis ${ }^{5}$, Douglas Campbell ${ }^{5}$, \\ Matthew Chan ${ }^{6}$, Rui Moreno ${ }^{7}$, Rupert M. Pearse ${ }^{8,9^{*}} \mathbb{D}$ and The International Surgical Outcomes Study (ISOS) \\ group
}

(c) 2017 Springer-Verlag Berlin Heidelberg and ESICM

\begin{abstract}
Purpose: As global initiatives increase patient access to surgical treatments, there is a need to define optimal levels of perioperative care. Our aim was to describe the relationship between the provision and use of critical care resources and postoperative mortality.

Methods: Planned analysis of data collected during an international 7-day cohort study of adults undergoing elective in-patient surgery. We used risk-adjusted mixed-effects logistic regression models to evaluate the association between admission to critical care immediately after surgery and in-hospital mortality. We evaluated hospital-level associations between mortality and critical care admission immediately after surgery, critical care admission to treat life-threatening complications, and hospital provision of critical care beds. We evaluated the effect of national income using interaction tests.
\end{abstract}

Results: 44,814 patients from 474 hospitals in 27 countries were available for analysis. Death was more frequent amongst patients admitted directly to critical care after surgery (critical care: 103/4317 patients [2\%], standard ward: 99/39,566 patients [0.3\%]; adjusted OR 3.01 [2.10-5.21]; $p<0.001$ ). This association may differ with national income (high income countries OR 2.50 vs. low and middle income countries OR 4.68; $p=0.07$ ). At hospital level, there was no association between mortality and critical care admission directly after surgery $(p=0.26)$, critical care admission to treat complications ( $p=0.33$ ), or provision of critical care beds $(p=0.70)$. Findings of the hospital-level analyses were not affected by national income status. A sensitivity analysis including only high-risk patients yielded similar findings.

Conclusions: We did not identify any survival benefit from critical care admission following surgery.

Keywords: Postoperative care/methods, Postoperative care/statistics and numerical data, Surgical procedures, operative/mortality, Critical care/utilisation

\footnotetext{
*Correspondence: r.pearse@qmul.ac.uk

${ }^{9}$ Adult Critical Care Unit, Royal London Hospital, London E1 1BB, UK

Full author information is available at the end of the article

Members of study group are listed in the supplementary file.
}

Take-home message: We analysed data from 44,814 patients in 27 countries to investigate the effect of postoperative critical care on survival. No evidence of survival benefit was found.

\section{县 Springer}




\section{Introduction}

The Commission on Global Surgery has set ambitious targets to improve patient access to surgical treatments [1]. Current estimates suggest that 310 million patients undergo surgery worldwide each year, with more procedures taking place in high income countries [2, 3]. Findings from epidemiological studies suggest that 4.8 billion people are unable to access safe surgical treatments [4], and the commission has recommended that at least 143 million additional procedures are required each year, primarily in low and middle income countries [5]. However, as healthcare systems develop to improve access to surgical treatments, and more complex procedures are offered, the number of patients who suffer postoperative complications will also increase $[1,4]$.

Estimates from high income countries suggest that postoperative complications occur in up to $20 \%$ of patients $[6,7]$, and short-term mortality may vary from 1 to $4 \%$ [8-16]. While effective perioperative care is considered essential to the safe provision of surgical treatments [17], the optimal level of such care has not been defined. Admission to a critical care unit is often considered necessary to prevent or treat life-threatening complications. However, this standard of patient care is very expensive, and there is little or no evidence to confirm the amount of critical care resource provision needed for a safe surgical service. The value of routine admission of highrisk patients to a critical care unit after surgery is hotly debated. In developed nations, there is some evidence that this resource is not allocated to patients at greatest need [8-10]. The findings of healthcare registry studies of postoperative critical care admission in the UK are complex and inconsistent $[18,19]$. Meanwhile, a study of Medicare data in the USA failed to identify any benefit of critical care admission after surgery $[20,21]$.

We therefore need to better understand the benefits of the provision and use of routine critical care admission after surgery. During the International Surgical Outcomes Study (ISOS) we collected patient- and hospital-level data describing critical care utilisation following elective surgery in 27 countries [22]. The aim of this prospective analysis was to describe the relationship between provision and use of critical care resources and subsequent postoperative mortality.

\section{Methods}

\section{Data collection}

This was a planned analysis of data collected during an international 7-day cohort study. The inclusion criteria were all adult patients (age $\geq 18$ years) undergoing elective surgery with a planned overnight stay in hospital. Patients undergoing emergency surgery, day-case surgery or radiological procedures were excluded. Each participating country selected a single data collection week between April and August 2014. The methods and basic epidemiological data from ISOS have been published elsewhere [22]. Regulatory requirements differed between countries with some requiring research ethics approval and some requiring only data governance approval. In the UK, the study was approved by the Yorkshire and Humber Research Ethics Committee (Reference: 13/YH/0371). Only hospitals returning valid data describing 20 or more patients, countries with ten or more participating hospitals, and patients with complete outcome data were eligible for inclusion in the main ISOS database. Data describing perioperative care facilities were collected for each hospital at the beginning of the study. Data describing patient outcomes were collected until hospital discharge on paper case record forms [23]. Data were censored at 30 days following surgery for patients who remained in hospital. Critical care was prospectively defined for all countries as a facility routinely capable of admitting patients who require invasive ventilation overnight. We did not attempt to standardise the admission criteria for postoperative critical care in participating hospitals.

\section{Objectives}

The objective of this analysis were to assess the association between provision and use of critical care resources and in-hospital mortality after elective surgery. We also investigated whether this association differed between high income countries (HIC) and low or middle income countries (LMIC). The potential effects of critical care were evaluated at the patient level and the hospital level adjusting for potential confounding factors. We initially performed a patient-level risk-adjusted analysis for patients admitted to a critical care unit immediately after surgery. In order to address potential problems with unmeasured confounding, we then performed three hospital-level analyses, evaluating hospital rates of critical care admission immediately after surgery, rates of critical care admission to treat life-threatening complications after surgery, and finally critical care capacity at the hospital level.

\section{Patient-level analysis of association between critical care admission and mortality}

We assessed the association between admission directly to critical care after surgery (versus admission to a standard ward) and in-hospital mortality using a mixed-effects logistic regression model with a random intercept for country [24]. The model was adjusted for the following baseline risk factors: age, gender, current smoker, American Society of Anesthesiologists physical status (ASA) score, severity of surgery (minor, intermediate, major), 
surgical procedure category and presence of ischaemic heart disease, heart failure, diabetes mellitus, chronic obstructive pulmonary disease/asthma, cirrhosis, stroke, and other co-morbid diseases. We adjusted for age using restricted cubic splines to account for a possible non-linear association with mortality [25]. We assessed whether the association between critical care admission and mortality differed according to a national income status (high income countries versus low and middle income countries) using an interaction test. As a result of low rates of missing data, we performed a complete case analysis which excluded patients with missing data describing baseline risk factors or critical care admission.

\section{Hospital-level analysis of mortality and critical care admission immediately after surgery}

We assessed the association between the hospital rates of critical care admission and hospital rate of risk-adjusted postoperative mortality. The risk-adjusted mortality rate for each hospital was calculated by first calculating the ratio of observed to expected deaths within each hospital. The number of expected deaths was calculated using a three-level mixed-effects logistic regression model with death as the outcome and random effects for country and hospital, adjusted for the same set of baseline risk factors as in the individual-level analysis above. We calculated the probability of death for each patient based on this model, and then calculated the expected number of deaths in each hospital by summing the probability for each patient within the hospital. We calculated the riskadjusted mortality rate by multiplying the observed to expected ratio for each hospital by the overall proportion of deaths in the dataset. We then used a linear regression model with the risk-adjusted mortality rate in each hospital as the outcome and critical care admission rate at the hospital level as a fixed factor. We modelled the association between critical care admission and risk-adjusted mortality using restricted cubic splines with three knots, placed at the percentiles as recommended by Harrell [25]. We weighted each observation according to the number of patients in that hospital. As above, we used an interaction test to assess whether the association between critical care admission and risk-adjusted mortality differed according to a national income status. We performed a complete case analysis excluding patients with missing data for baseline risk factors.

\section{Hospital-level analysis of mortality and critical care admission to treat a postoperative complication}

We assessed the association between critical care admission to treat a life-threatening postoperative complication and the risk-adjusted mortality rate at the hospital level using the same approach as above. We included the proportion of patients admitted to critical care to treat a postoperative complication in each hospital in the linear regression model as a fixed factor. We performed a complete case analysis excluding patients with missing data for baseline risk factors. We also excluded hospitals in which no patient experienced a postoperative complication, as it was impossible to calculate the proportion of patients admitted to critical care for this indication.

\section{Hospital-level analysis of association between mortality and critical care capacity}

We assessed the association between critical care capacity, defined as the number of critical care beds as a proportion of the total number of hospital beds (i.e. a critical care capacity of $5 \%$ would indicate that $5 \%$ of all hospital beds were critical care beds), and the risk-adjusted mortality rate at the hospital level using the same approach as above. We included critical care capacity in each hospital in the linear regression model as a fixed factor. We performed a complete case analysis excluding patients with missing data for baseline risk factors and hospitals with missing data on the number of beds. All analyses were performed using Stata 14 (StataCorp, USA).

\section{Sensitivity analyses}

We conducted two sensitivity analyses. In the first, we repeated each of the four main analyses described above in a high-risk subgroup, defined as patients classified as ASA III or IV who underwent major surgery. In the second, we investigated the possibility of different associations between critical care use and mortality in tertiary referral centres by repeating the three hospital-level analyses above using an interaction test for university vs. non-university hospitals.

\section{Results}

Patient data from 27 countries were included in this analysis. According to the World Bank classification [26], eight were classed as low or middle income countries (Brazil, China, Indonesia, Malaysia, Nigeria, Romania, South Africa, and Uganda) and 19 were classed as high income countries (Australia, Austria, Belgium, Canada, Denmark, France, Germany, Greece, Hong Kong, Italy, Netherlands, New Zealand, Portugal, Russia, Spain, Sweden, Switzerland, UK, and the USA). In total, 44,814 patients from 474 hospitals were included in the main database $(15,806$ patients from 126 hospitals in low or middle income countries, and 29,008 patients from 348 hospitals in high income countries) (Supplementary Fig. 1). Patient- and hospital-level characteristics are presented in Tables 1 and 2. Patients in low or middle income countries were younger, had lower ASA scores, and were less likely to have co-morbid disease. Patients admitted directly to 
Table 1 Baseline patient characteristics according to national income status (high vs. low and middle income countries), and level of care immediately following surgery (critical care vs standard hospital ward)

\begin{tabular}{|c|c|c|c|c|}
\hline & $\begin{array}{l}\text { Low and middle income } \\
\text { countries } \\
(n=15,806)\end{array}$ & $\begin{array}{l}\text { High income countries } \\
(n=29,008)\end{array}$ & $\begin{array}{l}\text { Admitted to critical care } \\
\text { after surgery } \\
\text { ( } n=4360)\end{array}$ & $\begin{array}{l}\text { Admitted to ward } \\
\text { after surgery } \\
(n=39,935)\end{array}$ \\
\hline Age (years) & $51(39,63)$ & $60(46,71)$ & $63(52,73)$ & $56(42,68)$ \\
\hline Male & $6931(44)$ & $13,527(47)$ & $2505(57)$ & $17,716(44)$ \\
\hline \multicolumn{5}{|l|}{ ASA score } \\
\hline I & $5104(32)$ & $6123(21)$ & $300(7)$ & $10,733(27)$ \\
\hline$\|$ & $8662(55)$ & $13,603(47)$ & $1244(29)$ & $20,806(52)$ \\
\hline III & $1849(12)$ & $8344(29)$ & $2256(52)$ & $7849(20)$ \\
\hline IV & $180(1)$ & $858(3)$ & $553(13)$ & $473(1)$ \\
\hline Metastatic cancer & $297(2)$ & $1409(5)$ & $316(7)$ & $1373(3)$ \\
\hline At least one co-morbidity & $6488(41)$ & $19,590(68)$ & 3551 (81) & $22,329(56)$ \\
\hline \multicolumn{5}{|l|}{ Surgical procedure } \\
\hline Orthopaedic & $2549(16)$ & $6910(24)$ & $329(8)$ & $9034(23)$ \\
\hline Breast & $622(4)$ & $916(3)$ & $22(1)$ & $1499(4)$ \\
\hline $\begin{array}{l}\text { Obstetrics and } \\
\text { gynaecology }\end{array}$ & $2213(14)$ & $3461(12)$ & $154(4)$ & $5466(14)$ \\
\hline Urology and kidney & $1632(10)$ & $3239(11)$ & $186(4)$ & $4644(12)$ \\
\hline Upper gastrointestinal & $709(4)$ & $1277(4)$ & $310(7)$ & $1653(4)$ \\
\hline Lower gastrointestinal & $974(6)$ & $2099(7)$ & $277(6)$ & $2757(7)$ \\
\hline Hepatobiliary & $1216(8)$ & $1066(4)$ & $207(5)$ & $2058(5)$ \\
\hline Vascular & $413(3)$ & $1186(4)$ & $255(6)$ & $1337(3)$ \\
\hline Head and neck & $2680(20)$ & $3830(13)$ & $632(15)$ & $5835(15)$ \\
\hline Plastics and cutaneous & $412(3)$ & $1258(4)$ & $51(1)$ & $1579(4)$ \\
\hline Cardiac & $294(2)$ & $1422(5)$ & $1488(34)$ & $228(1)$ \\
\hline Thoracic & $417(3)$ & $740(3)$ & $271(6)$ & $874(2)$ \\
\hline Other & $1671(11)$ & $1599(6)$ & $178(4)$ & $2968(7)$ \\
\hline
\end{tabular}

Data presented as median (IQR) or $n(\%)$

Table 2 Hospital- and patient-level characteristics for high vs. low and middle income country

\begin{tabular}{lcc} 
& Low and middle income countries & High income countries \\
\hline Hospital resources & & $570(361-835)$ \\
\hline Number of hospital beds & $825(412-1318)$ & $20(11-37)$ \\
\hline Number of critical care beds & $25(12-45)$ & $3.6 \%(2.4-5.9 \%)$ \\
\hline Critical care capacity & $2.8 \%(1.5-4.8 \%)$ & $5748(20 \%)$ \\
Critical care use & & $3271(11 \%)$ \\
\hline Number of patients with complication(s) & $1760(11 \%)$ & $878 / 5669(15 \%)$ \\
\hline Direct admission to critical care after surgery & $1046(7 \%)$ & $2.1(3.4)$ \\
\hline Critical care admission following a complication & $299 / 1749(17 \%)$ & $4.7(5.5)$ \\
\hline Length of hospital stay & & $2.8(4.5)$ \\
\hline Post-anaesthetic care unit stay (h) & $2.1(6.8)$ & $5.8(7.4)$ \\
\hline Hospital (days) & $6.6(5.6)$ & $2.3(3.5)$ \\
\hline Critical care stay for planned admissions (days) & $4.2(5.3)$ & \\
\hline Critical care stay to treat complications (days) & & \\
\hline
\end{tabular}

Data presented as mean (SD), median (IQR), or $n$ (\%). Critical care capacity is calculated as the ratio of critical care beds to the total number of hospital beds 
Table 3 Crude and risk-adjusted mortality for patients according to admission directly to critical care after surgery

\begin{tabular}{|c|c|c|c|c|}
\hline & $\begin{array}{l}\text { Mortality amongst patients } \\
\text { admitted to standard ward } \\
\text { after surgery }(n=39,566)\end{array}$ & $\begin{array}{l}\text { Mortality amongst patients } \\
\text { admitted to critical care } \\
\text { after surgery }(n=4317)\end{array}$ & $\begin{array}{l}\text { Risk-adjusted odds ratio } \\
(95 \% \mathrm{CI})\end{array}$ & $p$ value for interaction \\
\hline Overall & $99(0.3 \%)$ & $103(2.4 \%)$ & $3.01(2.10-5.21)$ & - \\
\hline $\begin{array}{l}\text { Low and middle income } \\
\text { countries }\end{array}$ & $25(0.2 \%)$ & $29(2.8 \%)$ & $4.68(2.56-8.57)$ & - \\
\hline High income countries & $74(0.3 \%)$ & $74(2.3 \%)$ & $2.50(1.65-3.78)$ & 0.07 \\
\hline
\end{tabular}

Data presented as $n(\%)$ and odds ratios (OR). OR were estimated using mixed-effects logistic regression models, with a random intercept for country. Patients with missing baseline covariates or with missing data on critical care admission after surgery were excluded from the analysis. In total, $43,883 / 44,814$ (98\%) patients were included in the analysis. $p$ value for interaction assesses whether the OR differs between low and middle income countries and high income countries

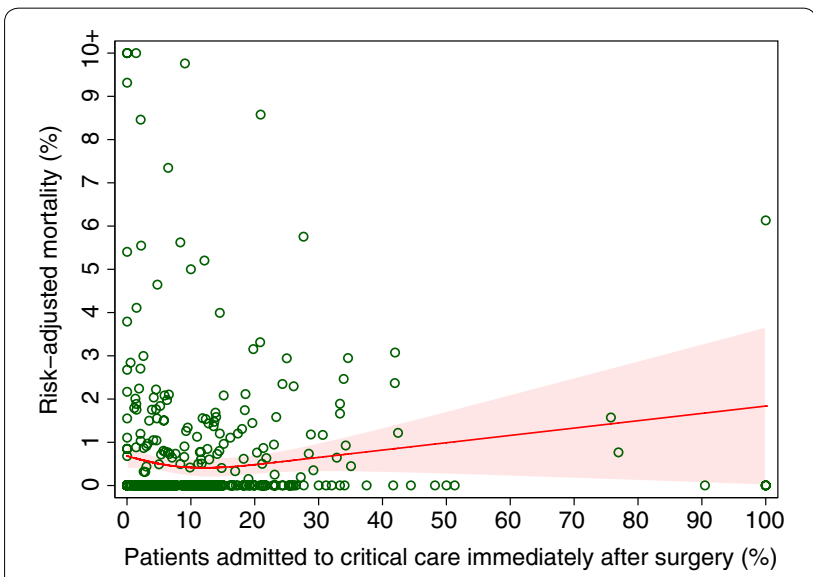

Fig. 1 Risk-adjusted mortality following in-patient elective surgery in 469 hospitals across 27 countries according to proportion of patients admitted to critical care immediately after surgery. Each data point represents one hospital. The association between the proportion of patients admitted to critical care immediately after surgery and risk-adjusted mortality was estimated at the hospital level using a linear regression model with the risk-adjusted mortality rate in each hospital as the outcome and the proportion of patients admitted to critical care immediately after surgery in each hospital as a covariate. The proportion of patients admitted to critical care immediately after surgery was modelled using restricted cubic splines. For greater clarity of presentation, hospitals with greater than $10 \%$ risk-adjusted mortality rate have been censored at 10\% in the graph; however, these values were not censored in the linear regression model used to estimate the association critical care after surgery were older, had higher ASA scores, and were more likely to have co-morbid disease. Hospitals in high income countries had higher levels of critical care capacity and direct admission to critical care after surgery. Ninety hospitals did not admit any patients to critical care directly after surgery (19\%), 32 in low or middle income countries and 58 in high income countries. A total of 134 hospitals did not admit any patients to critical care to treat a complication, 37 in low or middle income countries and 97 in high income countries.

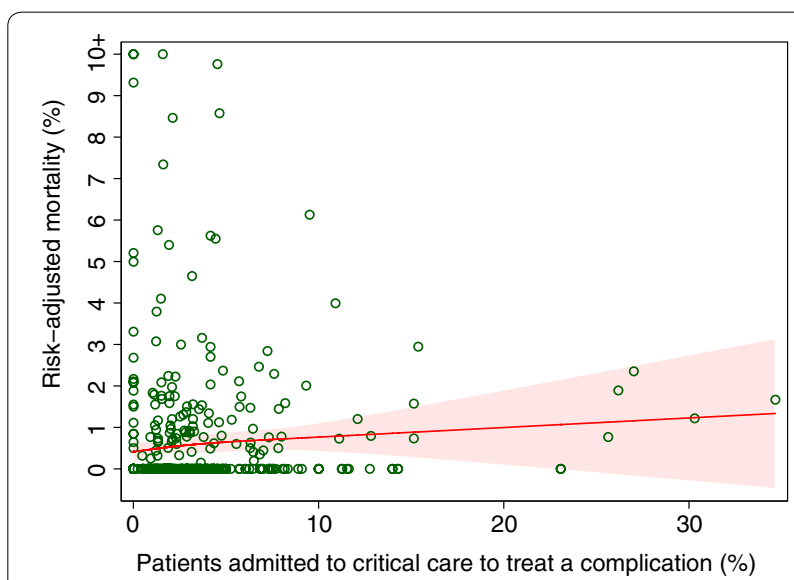

Fig. 2 Risk-adjusted mortality following in-patient elective surgery in 449 hospitals across 27 countries according to proportion of patients admitted to critical care to treat a life-threatening postoperative complication. Each data point represents one hospital. The association between the proportion of patients admitted to critical care to treat a complication and risk-adjusted mortality was estimated at the hospital level using a linear regression model with the risk-adjusted mortality rate in each hospital as the outcome and the proportion of patients admitted to critical care to treat a complication in each hospital as a covariate. The proportion of patients admitted to critical care to treat a complication was modelled using restricted cubic splines. For greater clarity of presentation, hospitals with greater than $10 \%$ risk-adjusted mortality rate have been censored at 10\% in the graph. However, these values were not censored in the linear regression model used to estimate the association

\section{Patient-level analysis of association between critical care admission and mortality}

Overall, 43,883 patients (98\%) were included in the analysis (Table 3). Patients admitted to critical care directly after surgery had a higher mortality rate $(2.4 \%)$ than patients admitted to a standard ward (0.3\%). After risk adjustment, the odds ratio for mortality was 3.01 (95\% confidence intervals $2.10-5.21 ; p<0.001)$. There was some evidence that this association differed according to a national income status ( $p$ value for interaction 0.07 ). 


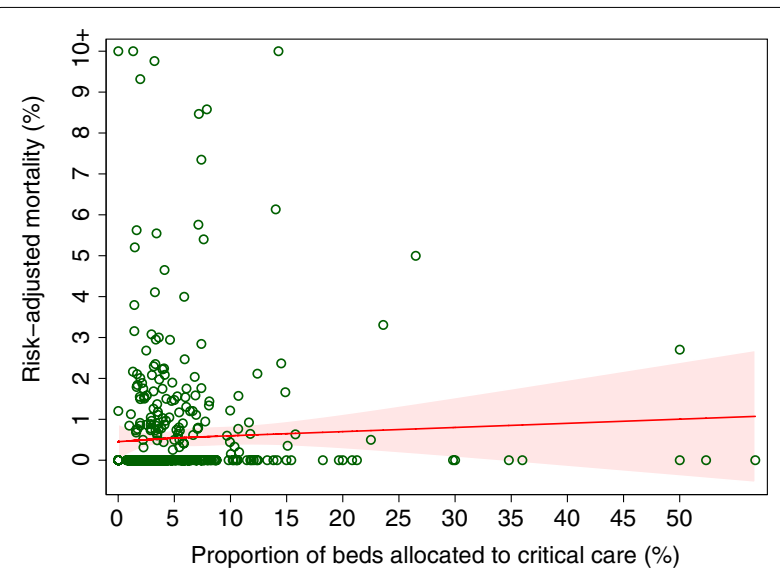

Fig. 3 Risk-adjusted mortality following in-patient elective surgery in 468 hospitals across 27 countries according to number of critical care beds as a proportion of total hospital beds. Each data point represents one hospital. The association between critical care beds and risk-adjusted mortality was estimated at the hospital level using a linear regression model with the risk-adjusted mortality rate in each hospital as the outcome and the proportion of critical care beds in each hospital as a covariate. The proportion of critical care beds was modelled using restricted cubic splines. For greater clarity of presentation, hospitals with greater than $10 \%$ risk-adjusted mortality rate have been censored at 10\% in the graph. However, these values were not censored in the linear regression model used to estimate the association

\section{Hospital-level analysis of mortality and critical care admission immediately after surgery}

Overall, 44,363 patients (99\%) from 469 hospitals (99\%) were included in the analysis. We found no association between critical care admission immediately after surgery and mortality ( $p=0.26$ ) (Fig. 1$)$. We found no evidence that this association differed between low or middle countries and high income countries ( $p$ value for interaction 0.27).

\section{Hospital-level analysis of mortality and critical care admission to treat a postoperative complication}

Overall, 43,771 patients (98\%) from 449 hospitals (95\%) were included in the analysis. We found no association between critical care admission to treat a postoperative complication and mortality ( $p=0.33$ ) (Fig. 2). We found no evidence that this association differed between low or middle and high income countries ( $p$ value for interaction 0.98).

\section{Hospital-level analysis of association between mortality and critical care capacity}

Overall, 44,342 patients (99\%) from 468 hospitals (99\%) were included in the analysis. We found no association between critical care capacity and mortality $(p=0.70)$ (Fig. 3). We found no evidence that this association differed between low or middle and high income countries ( $p$ value for interaction 0.43 ).

\section{Sensitivity analyses \\ High-risk subgroup}

A total of 5624 patients were defined as high risk (classified as ASA III or IV and undergoing major surgery), of whom 5550 (99\%) were included in the analysis. The overall mortality rate was $2 \%(n=109)$. The risk-adjusted odds ratio for admission to critical care immediately after surgery was 2.32 (95\% CI 1.44-3.74). We found no significant effect of any critical care measure in the high-risk subgroup of patients (Supplementary Figs. 2-4).

\section{Tertiary referral centres}

At the hospital level, there was some evidence that the relationship between critical care admission immediately after surgery and mortality varied according to hospital type ( $p$ value for interaction 0.05 ), although there was no benefit of increasing rates of critical care admission in either subgroup. We found no evidence that university hospital status modified the relationship between postoperative mortality and critical care admission to treat a postoperative complication ( $p$ value for interaction 0.84 ) or critical care capacity ( $p$ value for interaction 0.88 ).

\section{Discussion}

The principal finding of this analysis was that we were not able to identify any evidence of patient survival benefit from admission to critical care immediately following surgery. At the patient level, mortality rates remained higher amongst patients admitted directly to critical care even after risk adjustment, although this observation seems certain to be affected by significant residual confounding. However, our hospital-level analyses, which are much less sensitive to this form of bias, identified no protective effect from increasing the provision or use of critical care. We conducted post hoc sensitivity analyses to explore the effect of postoperative critical care admission within a high-risk subgroup of patients and according to university hospital status. However, we did not identify a protective effect of critical care in either of these analyses. We would urge caution in the interpretation of these findings. The most effective hospital care pathways will not be based purely on high rates of postoperative critical care admission, but it remains unclear how provision of this resource should change, in particular for high-risk patients.

The value of routine admission of high-risk patients to critical care after surgery is debated. There is evidence of widespread inequity in the allocation of this resource, leading to a sustained interest in preoperative risk assessment [27]. For example, admission to critical care after cardiac surgery is routine in most countries, 
whilst high-risk patient groups undergoing non-cardiac surgery are often not provided with this level of care despite a much higher mortality rate $[8,11,12]$. Overall rates of postoperative admission to critical care remain low, even amongst high-risk patients [8-10]. The findings of an analysis of a large US Medicare dataset exploring the association between mortality, length of hospital stay, and healthcare costs with critical care admission for patients over 65 years of age undergoing one of five major surgical procedures did not identify any benefit of critical care admission $[20,21]$. In a recent healthcare registry study within the UK, investigators identified significant regional variations in postoperative mortality. After adjusting for regional variations in the provision and utilisation of postoperative critical care, the number of unexplained postoperative deaths decreased [18]. An analysis of emergency surgical admissions in England identified wide variations in crude mortality, which appeared to be lower in those hospitals with the highest levels of medical and nursing staffing and critical care beds relative to size [19].

It seems counter-intuitive that we did not identify a clear beneficial effect of critical care admission on patient outcomes in this analysis. Whilst the most obvious explanation may simply be that critical care admission is ineffective, there are several other explanations for this observation. As it is impossible to test the benefits of critical care admission in a classic individual patient randomised trial, we must use analyses of large datasets to perform a "natural trial", comparing outcomes for similar patients allocated to different standards of care as part of their normal treatment. The major challenge of this approach is to understand enough about each patient to allow robust statistical adjustment for baseline risk. If baseline data fail to describe this risk, then important differences between patients are not accounted for and unmeasured confounding results. It is difficult to precisely adjust for risk in a mixed surgical population. Key risk factors for patients undergoing one type of surgery may not be applicable to others. Cancer surgery is an important example, where tumour invasion may result in wide variation in risk, even between patients having the same procedure. Residual confounding due to unknown variables or interactions in our risk adjustment models seems the likely explanation for the apparent excess mortality associated with critical care admission in our patient-level analysis. Another important factor influencing the apparent benefit of critical care is the incremental value over care on a standard ward. Historically, critical care units were developed to provide organ support for patients with life-threatening illness. However, few surgical patients require such treatments immediately after surgery. The initial problems which most frequently impede postoperative recovery in high-risk patients include pain, hypothermia, mild cardiorespiratory compromise, fluid imbalance, and nutrition [17]. These problems can often be effectively tackled on a standard ward through proactive patient care led by experienced qualified nursing staff, with prompt access to medical support when required. Adequate staffing of surgical wards with qualified nurses may improve patient safety $[28,29]$ and reduce the incidence of pneumonia, surgical site infection, and postoperative sepsis [30-32]. Hospitals with higher nurse staffing levels may also be more cost-effective in terms of postoperative outcomes (lower mortality with similar costs), especially for high-risk surgical patients [33]. Thus in hospitals which deliver excellent ward-based care, perhaps through provision of intermediate care wards, the incremental benefit of critical care admission will be reduced. One additional explanation may be that critical care resources are not allocated to the surgical patients at greatest risk of death because of inadequate risk assessment, or failure of the methods used for doing so $[8,9,11,12]$, thereby reducing the effectiveness of the treatment.

The strengths of this study include the large number of consecutive patients enrolled worldwide, using prospective standardised definitions for critical care. We also distinguished admission directly to critical care after elective surgery from subsequent admission to treat postoperative complications. Despite our concerns regarding residual confounding, we collected more detailed data on baseline risk factors than would be available in most large healthcare registries. Patient-level variables were selected on the basis that they were objective, routinely collected for clinical reasons, could be transcribed with a high level of accuracy, and would be relevant to a risk adjustment model which included a wide variety of surgical procedures. The study also has a number of weaknesses. Despite the large sample size, we cannot consider this study as representative of current practice in all countries. In order to maximise the quantity and quality of data collected, we only recruited patients undergoing elective surgery, and our findings cannot be extrapolated to postoperative care for patients undergoing emergency procedures. Only a small proportion of hospitals took part in a small number of countries. Many patients were enrolled in university hospitals whilst smaller, low volume centres are under-represented. This may be more important for the low and middle income countries which took part. The risk adjustment methods used may not fully account for high mortality rates in hospitals specialising in more complex surgery. Our findings may also be limited by the low event rate for mortality. Although we collected very detailed data on the process of postoperative care, we cannot fully describe what happened to every 
patient. For example, we do not know how many patients were admitted to critical care because of unexpected complications during surgery, and we do not know how many patients were not admitted to critical care because of a lack of available beds. It was beyond the scope of this study to attempt to standardise the admission criteria for postoperative critical care. Finally, although we planned to enrol every eligible patient undergoing surgery during the study period, we cannot be sure of the exact proportion of eligible patients included.

\section{Conclusions}

In this prospective analysis of data from an international cohort study, we did not identify any survival benefit from postoperative admission to critical care, either at the patient level or the hospital level. Safe perioperative care remains essential for effective surgical treatment. However, isolated measures to increase postoperative admission to critical care may not alone be sufficient to reduce mortality. We would urge caution in the interpretation of these findings. It remains unclear how provision of this resource should change, in particular for high-risk surgical patients.

\section{Electronic supplementary material}

The online version of this article (doi:10.1007/s00134-016-4633-8) contains supplementary material, which is available to authorized users.

\section{Author details}

${ }^{1}$ Queen Mary University of London, London E1 2AT, UK. ${ }^{2}$ Attikon University Hospital, Athens, Greece. ${ }^{3}$ University of Queensland, Brisbane, Australia. ${ }^{4}$ Papageorgiou General Hospital, Thessaloniki, Greece. ${ }^{5}$ Auckland City Hospital, Auckland, New Zealand. ${ }^{6}$ Chinese University of Hong Kong, Sha Tin, Hong Kong. ${ }^{7}$ Hospital de São José, Centro Hospitalar de Lisboa Central, Lisboa, Lisbon, Portugal. ${ }^{8}$ Queen Mary University of London, London EC 1 M 6BQ, UK. ${ }^{9}$ Adult Critical Care Unit, Royal London Hospital, London E1 1BB, UK.

\begin{abstract}
Acknowledgements
This was an investigator initiated study funded by Nestle Health Sciences through an unrestricted research grant, and by a National Institute for Health Research (UK) Professorship held by RP. The study was sponsored by Queen Mary University of London. ISOS investigators were entirely responsible for study design, conduct, and data analysis. The authors had full data access and were solely responsible for data interpretation, drafting and critical revision of the manuscript, and the decision to submit for publication.
\end{abstract}

\section{Data sharing}

The authors are happy to consider data sharing requests from bona fide researchers. Participant consent was not obtained but the presented data are anonymised and risk of identification is low. Enquiries should be addressed to the chief investigator at admin@isos.org.uk.

\section{Compliance with ethical standards}

\section{Conflicts of interest}

$\mathrm{RP}$ holds research grants and has given lectures and/or performed consultancy work for Nestle Health Sciences, BBraun, Medtronic, and Edwards Lifesciences, and is a member of the Associate Editorial Board of the British Journal of Anaesthesia. All other authors declare they have no conflicts of interest.

Received: 18 September 2016 Accepted: 21 November 2016 Published online: 25 April 2017

\section{References}

1. The Lancet commission on global surgery (2016) http://www.lancetglobalsurgery.org/. Accessed 3 Nov 2016

2. Weiser TG, Regenbogen SE, Thompson KD, Haynes AB, Lipsitz SR, Berry WR, Gawande AA (2008) An estimation of the global volume of surgery: a modelling strategy based on available data. Lancet 372:139-144

3. Weiser TG, Haynes AB, Molina G, Lipsitz SR, Esquivel M, Uribe-Leitz T, Fu R, Azad T, Chao TE, Berry WR, Gawande AA (2015) Estimate of the global volume of surgery in 2012: an assessment supporting improved health outcomes. Lancet 385(Suppl 2):S11

4. Alkire BC, Raykar NP, Shrime MG, Weiser TG, Bickler SW, Rose JA, Nutt CT, Greenberg SL, Kotagal M, Riesel JN, Esquivel M, Uribe-Leitz T, Molina G, Roy N, Meara JG, Farmer PE (2015) Global access to surgical care: a modelling study. Lancet Glob Health 3:e316-323

5. Rose J, Weiser TG, Hider P, Wilson L, Gruen RL, Bickler SW (2015) Estimated need for surgery worldwide based on prevalence of diseases: a modelling strategy for the WHO Global Health Estimate. Lancet Glob Health 3:S13-20

6. Ghaferi AA, Birkmeyer JD, Dimick JB (2009) Variation in hospital mortality associated with inpatient surgery. New Engl J Med 361:1368-1375

7. Khuri SF, Daley J, Henderson W, Hur K, Demakis J, Aust JB, Chong V, Fabri PJ, Gibbs JO, Grover F, Hammermeister K, Irvin G 3rd, McDonald G, Passaro E Jr, Phillips L, Scamman F, Spencer J, Stremple JF (1998) The Department of Veterans Affairs' NSQIP: the first national, validated, outcome-based, risk-adjusted, and peer-controlled program for the measurement and enhancement of the quality of surgical care. National VA Surgical Quality Improvement Program. Ann Surg 228:491-507

8. Pearse RM, Harrison DA, James P, Watson D, Hinds C, Rhodes A, Grounds RM, Bennett ED (2006) Identification and characterisation of the high-risk surgical population in the United Kingdom. Crit Care 10:R81

9. Pearse RM, Moreno RP, Bauer P, Pelosi P, Metnitz P, Spies C, Vallet B, Vincent $J$, Hoeft A, Rhodes A, European Surgical Outcomes Study group for the trials groups of the European Society of Intensive Care Medicine and the European Society of Anaesthesiology (2012) Mortality after surgery in Europe: a 7 day cohort study. Lancet 380:1059-1065

10. Gillies MA (2016) Intensive care utilisation and outcomes after high-risk surgery in Scotland: a population based cohort study. Brit J Anaesth. doi:10.1093/bja/aew396

11. Findlay G, Goodwin A, Protopappa K, Smith N, Mason M (2011) Knowing the risk: a review of the peri-operative care of surgical patients. National Confidential Enquiry into Patient Outcome and Death, London

12. Jhanji S, Thomas B, Ely A, Watson D, Hinds CJ, Pearse RM (2008) Mortality and utilisation of critical care resources amongst high-risk surgical patients in a large NHS trust. Anaesthesia 63:695-700

13. Glance LG, Lustik SJ, Hannan EL, Osler TM, Mukamel DB, Qian F, Dick AW (2012) The surgical mortality probability model: derivation and validation of a simple risk prediction rule for noncardiac surgery. Ann Surg 255:696-702

14. Jencks SF, Williams MV, Coleman EA (2009) Rehospitalizations among patients in the Medicare fee-for-service program. New Engl J Med 360:1418-1428

15. Noordzij PG, Poldermans D, Schouten O, Bax JJ, Schreiner FA, Boersma E (2010) Postoperative mortality in the Netherlands: a population-based analysis of surgery-specific risk in adults. Anesthesiology 112:1105-1115

16. Yu PC, Calderaro D, Gualandro DM, Marques AC, Pastana AF, Prandini JC, Caramelli B (2010) Non-cardiac surgery in developing countries: epidemiological aspects and economical opportunities-the case of Brazil. PLoS One 5:e10607

17. Pearse RM, Holt PJ, Grocott MP (2011) Managing perioperative risk in patients undergoing elective non-cardiac surgery. BMJ 343:d5759

18. Gillies MA, Power GS, Harrison DA, Fleming A, Cook B, Walsh TS, Pearse RM, Rowan KM (2015) Regional variation in critical care provision and outcome after high-risk surgery. Intensive Care Med 41:1809-1816

19. Ozdemir BA, Sinha S, Karthikesalingam A, Poloniecki JD, Pearse RM, Grocott MP, Thompson MM, Holt PJ (2016) Mortality of emergency general surgical patients and associations with hospital structures and processes. Br J Anaesth 116:54-62

20. Wunsch H, Gershengorn HB, Cooke CR, Guerra C, Angus DC, Rowe JW, Li $\mathrm{G}$ (2016) Use of intensive care services for medicare beneficiaries undergoing major surgical procedures. Anesthesiology 124(4):899-907 
21. Gillies MA, Pearse RM (2016) Intensive care after high-risk surgery: what's in a name? Anesthesiology 124(4):761-762

22. International Surgical Outcomes Study Group (2016) Global patient outcomes after elective surgery: prospective cohort study in 27 low, middle and high income countries. Br J Anaesth 117(5):601-609

23. Jammer I, Wickboldt N, Sander M, Smith A, Schultz MJ, Pelosi P, Leva B, Rhodes A, Hoeft A, Walder B, Chew MS, Pearse RM (2015) Standards for definitions and use of outcome measures for clinical effectiveness research in perioperative medicine: European Perioperative Clinical Outcome (EPCO) definitions: a statement from the ESA-ESICM joint taskforce on perioperative outcome measures. Eur J Anaesthesiol 32(2):88-105

24. Rabe-Hesketh S, Skrondal A (2012) Multilevel and longitudinal modeling using stata. Stata, Texas

25. Harrell FE (2001) Regression modeling strategies: with applications to linear models, logistic regression, and survival analysis. Springer, New York

26. World Bank (2016) Classification of national income. http://www.worldbank.org/en/country. Accessed 3 Nov 2016

27. James S, Jhanji S, Smith A, O'Brien G, Fitzgibbon M, Pearse RM (2014) Comparison of the prognostic accuracy of scoring systems, cardiopulmonary exercise testing, and plasma biomarkers: a single-centre observational pilot study. Br J Anaesth 112:491-497
28. Griffiths P, Ball J, Drennan J, James L, Jones J, Recio-Saucedo A, Simon $M$ (2014) The association between patient safety outcomes and nurse/ healthcare assistant skill mix and staffing levels and factors that may influence staffing requirements. National Institute for Health and Care Excellence, London

29. Aiken LH, Clarke SP, Cheung RB, Sloane DM, Silber JH (2003) Educational levels of hospital nurses and surgical patient mortality. JAMA 290:1617-1623

30. Blegen MA, Goode CJ, Spetz J, Vaughn T, Park SH (2011) Nurse staffing effects on patient outcomes: safety-net and non-safety-net hospitals. Med Care 49:406-414

31. Cho SH, Ketefian S, Barkauskas VH, Smith DG (2003) The effects of nurse staffing on adverse events, morbidity, mortality, and medical costs. Nurs Res 52:71-79

32. McGillis Hall L, Doran D, Pink GH (2004) Nurse staffing models, nursing hours, and patient safety outcomes. J Nurs Adm 34:41-45

33. Silber JH, Rosenbaum PR, McHugh MD, Ludwig JM, Smith HL, Niknam BA Even-Shoshan O, Fleisher LA, Kelz RR, Aiken LH (2016) Comparison of the value of nursing work environments in hospitals across different levels of patient risk. JAMA Surg 151:527-536 